\title{
MS097.P01
}

\section{New iridates and ruthenates in garnet-like crystal-structures}

\author{
Thomas Doert $^{1}$, Hagen Poddig ${ }^{1}$, Jens Hunger ${ }^{1}$ \\ ${ }^{1}$ Dresden University Of Technology, Dresden, Germany \\ E-mail: thomas.doert@tu-dresden.de
}

Compounds of the second- and third-row late transition metals are currently under investigation due to their strong spinorbit coupling and the resulting physical properties [1]. Some new iridates and ruthenates with interesting properties could recently be prepared by hydrothermal synthesis $[2,3]$.

Attempts to synthesize new ruthenates and iridates in high oxidation states require a strongly oxidizing environment. We therefore used alkaline hydrigen peroxide solutions as reaction media for the oxidation of $M$ (III) ( $M=$ Ru, Ir) starting materials under hydrothermal conditions. The control of the oxidation states of the metal species in solution is important for the formation of pure products. The grown crystallites from the synthesis were large enough to determine the crystal structures with the general formula $\mathrm{Ca}_{3} \mathrm{Fe}_{3+x-\delta} \mathrm{M}_{2-x} \mathrm{O}_{12-y}(\mathrm{OH})_{y}$ by single crystal diffraction (Fig. 1). The Ru compound adopts the garnet structure in space group Ia $\overline{3 d}$, No. 230, with a lattice parameter of $a=12.489(1) \AA$ and $Z=8$. The octahedral site (Wyckoff site 16a) is mixed occupied by $\mathrm{Ru}(\mathrm{IV})(52 \%)$ and $\mathrm{Fe}$ (II) (48\%), whereas the tetrahedral site (24d) is

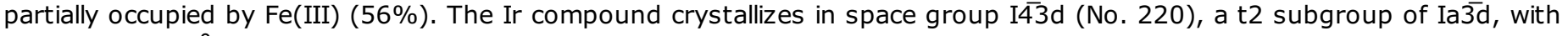
$a=12.540(1) \AA$, where the octahedral site is mixed occupied by $\operatorname{Ir}(\mathrm{IV})(69 \%)$ and Fe(II) ( $31 \%$ ). The symmetry reduction splits the $24 \mathrm{~d}$ site of the garnet in two non-equivalent tetrahedral sites, $12 \mathrm{a}$ and $12 \mathrm{~b}$, which are both partially occupied by $\mathrm{Fe}$ (III) ( $72 \%$ and $18 \%$, respectively) in the Ir compound.

Due to the partially occupied tetrahedral sites in both compounds, the presence of hydroxide ions was assumed for charge balancing. The presence of hydrogen in the compounds was confirmed by IR-spectroscopy and the hydrogen-content was quantified by carrier gas hot extraction. The local structure of the Fe cations evidenced by Mössbauer spectroscopy is in good agreement with the structure model obtained from diffraction data. Magnetic measurements indicate a complex magnetic behavior of the compounds with strong ferromagnetic coupling at low temperatures.

[1] W. Witczak-Krempa, G. Chen, Y. B. Kim, L. Balents, Annu. Rev. Condens. Matter Phys. 2014, 5, 57-82.

[2] K. Sardar, J. Fisher, D. Thompsett, M. R. Lees, G. J. Clarkson, J. Sloan, R. J. Kashtiban, R. I. Walton, Chemical Science $2011,2,1573$.

[3] C. I. Hiley, M. R. Lees, J. M. Fisher, D. Thompsett, S. Agrestini, R. I. Smith, R. I. Walton, Angew. Chem. Int. Ed. 2014, $53,4423-4427$.
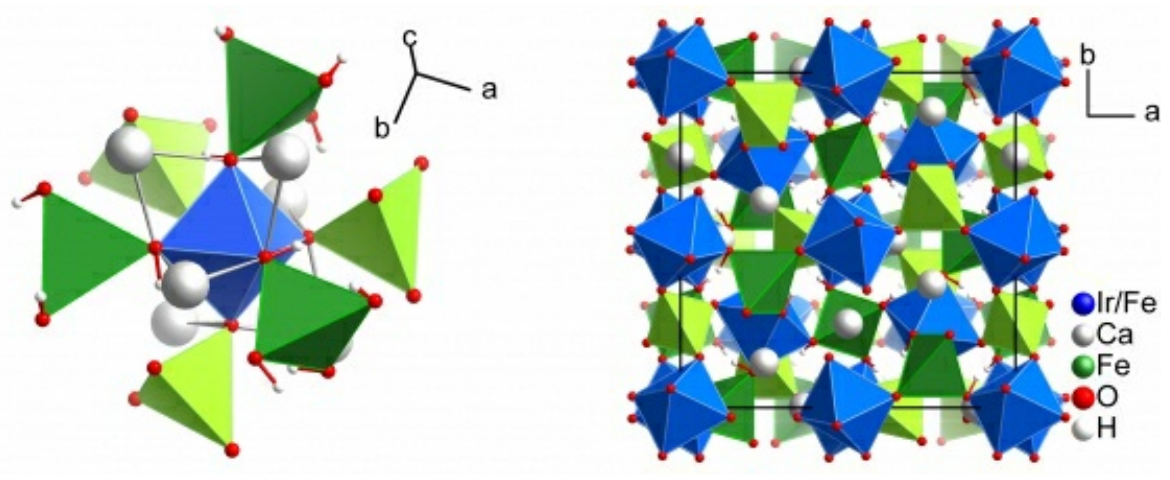

Keywords: garnets, hydrothermal synthesis 